\title{
Speed of Sound in Periodic Elastic Composites
}

\author{
A. A. Krokhin, ${ }^{1,2}$ J. Arriaga, ${ }^{2}$ and L. N. Gumen ${ }^{3}$ \\ ${ }^{1}$ Department of Physics, University of North Texas, P.O. Box 311427, Denton, Texas 76203, USA \\ ${ }^{2}$ Instituto de Física, Universidad Autónoma de Puebla, Apartado Postal J-48, Puebla, 72570 Mexico \\ ${ }^{3}$ Universidad Popular Autónoma del Estado de Puebla, Puebla, 72160 Mexico
}

(Received 6 September 2003; published 29 December 2003)

\begin{abstract}
We consider the low-frequency limit (homogenization) for propagation of sound waves in periodic elastic medium (phononic crystals). Exact analytical formulas for the speed of sound propagating in a three-dimensional periodic arrangement of liquid and gas or in a two-dimensional arrangement of solids are derived. We apply our formulas to the well-known phenomenon of the drop of the speed of sound in mixtures. For air bubbles in water we obtain a perfect agreement with the recent results of coherent potential approximation obtained by M. Kafesaki, R. S. Penciu, and E. N. Economou [Phys. Rev. Lett. 84, 6050 (2000)] if the filling of air bubbles is far from close packing. When air spheres almost touch each other, the approximation gives 10 times lower speed of sound than the exact theory does.
\end{abstract}

DOI: $10.1103 /$ PhysRevLett.91.264302

PACS numbers: $43.20 .+\mathrm{g}, 43.58 .+\mathrm{z}, 43.90 .+\mathrm{v}$

Acoustic waves play an important role in our life, being a universal carrier of information in vivid nature. In most cases, the media where the acoustic waves propagate are inhomogeneous. A common example is a mixture of water and air. During the past century the problem of propagation of sound in heterogeneous media has been extensively studied [1]. In the last few years artificial periodic elastic structures, phononic crystals, have been successfully fabricated [2]. Because of the presence of acoustic gaps - regions of frequencies where sound does not propagate [3] - phononic crystals can be used as soundless background for many technological devices. Phononic crystal with point, linear, and surface defects permit manipulation of sound: they guide the acoustic waves, split, and bend them [4]. At low frequencies phononic crystals possess a property to focus a sound beam [5] and thus may find numerous applications in acoustic surgery [6]. To design an acoustic lens one needs to know the refractive index of the material or the effective speed of sound. At low frequencies (well below the band gap) the dispersion relation is linear, $\omega=c_{\text {eff }} k$, since one wavelength covers many periods of the structure, thus averaging the inhomogeneous medium. Calculation of the effective parameters of the uniform medium (effective speed of sound, $c_{\text {eff }}$, and the effective elastic moduli) is a long-standing problem of the mathematical theory of homogenization [7]. Although the theory itself is well developed and predicts that different media homogenize at low frequencies, there are no explicit formulas that can be used for calculation of the effective parameters (see recent review [8] on homogenization for different types of the wave equations). There are also approximate methods that allow calculations of the effective parameters for particular structures [9-11]. Sometimes the effective elastic moduli can be evaluated from the exact upper and lower bounds [12].
In this Letter we develop an exact analytical theory of homogenization of periodic elastic structures. Our approach is based on the plane wave method that has been successfully used for homogenization of periodic dielectric medium (photonic crystals) [13]. We obtain compact formulas for $c_{\text {eff }}$ that take into account the multiple scattering and the microstructure of the periodic medium.

We start by considering a periodic arrangement of gas bubbles in a liquid. In the liquid-gas mixture the propagating mode is a longitudinal one, and it is characterized by a scalar parameter, e.g., pressure, $p(\mathbf{r})$, that satisfies the wave equation

$$
\frac{1}{\lambda(\mathbf{r})} \frac{\partial^{2} p}{\partial t^{2}}=\nabla \cdot\left(\frac{\nabla p}{\rho(\mathbf{r})}\right)
$$

where $\rho(\mathbf{r})$ and $\lambda(\mathbf{r})$ are the density and the local compressibility coefficient. In a periodic structure $1 / \lambda(\mathbf{r})$, $1 / \rho(\mathbf{r})$, and $p(\mathbf{r})$ can be expanded in a basis of plane waves,

$$
\begin{aligned}
\frac{1}{\lambda(\mathbf{r})} & =\sum_{\mathbf{G}} \gamma(\mathbf{G}) \exp (i \mathbf{G} \cdot \mathbf{r}), \\
\frac{1}{\rho(\mathbf{r})} & =\sum_{\mathbf{G}} \nu(\mathbf{G}) \exp (i \mathbf{G} \cdot \mathbf{r}), \\
p(\mathbf{r}, t) & =\exp (i \mathbf{k} \cdot \mathbf{r}-i \omega t) \sum_{\mathbf{G}} p_{k}(\mathbf{G}) \exp (i \mathbf{G} \cdot \mathbf{r}),
\end{aligned}
$$

where $\mathbf{k}$ and $\mathbf{G}$ are the Bloch vector and the reciprocal lattice vectors, respectively. Substituting Eq. (2) into Eq. (1) we get a generalized eigenvalue problem in $\mathbf{G}$ space,

$$
\begin{aligned}
\sum_{\mathbf{G}^{\prime}} \nu\left(\mathbf{G}-\mathbf{G}^{\prime}\right)(\mathbf{k} & +\mathbf{G}) \cdot\left(\mathbf{k}+\mathbf{G}^{\prime}\right) p_{k}\left(\mathbf{G}^{\prime}\right) \\
& =\omega^{2} \sum_{\mathbf{G}^{\prime}} \gamma\left(\mathbf{G}-\mathbf{G}^{\prime}\right) p_{k}\left(\mathbf{G}^{\prime}\right) .
\end{aligned}
$$


The dispersion relation $\omega=\omega_{n}(\mathbf{k})(n=1,2, \ldots)$ is obtained from the condition that the set of Eqs. (3) has a nontrivial solution.

It is easy to get from Eq. (3) that in the static limit, $\omega=k=0$, all Fourier coefficients $p_{k}(\mathbf{G} \neq 0)$ vanish. The only nonvanishing component $p_{k}(\mathbf{G}=0)=p_{0}$ determines the uniform static pressure $p_{0}$. Then the term with $\mathbf{G}=0$ gives the principal contribution to the Fourier expansion (2) of $p(\mathbf{r})$ in the long-wavelength limit,

$$
p(\mathbf{r}) \approx p_{0} \exp (i \mathbf{k} \cdot \mathbf{r})+\sum_{\mathbf{G} \neq 0} p_{k}(\mathbf{G}) \exp (i \mathbf{G} \cdot \mathbf{r})
$$

Equation (4) means that the medium homogenizes; i.e., the solution of the wave Eq. (1) approaches the plane wave when $k \rightarrow 0$. The sum over $\mathbf{G}$ vanishes linearly with $k$.

The effective speed of sound is obtained from Eqs. (3) and (4) by taking the limit

$$
c_{\text {eff }}(\hat{\mathbf{k}})=\lim _{k \rightarrow 0}(\omega / k) .
$$

It turns out to be dependent not only on the average characteristics of the periodic structure but on the details and geometry of the unit cell and on the direction of propagation $\hat{\mathbf{k}}=\mathbf{k} / k$. Equation (5) determines the phase velocity, which in the long-wavelength limit coincides with the group velocity since the dispersion is linear. We substitute now Eq. (4) into Eq. (3) and obtain two equations for the terms of the order of $k$ and $k^{2}$, respectively,

$$
\begin{gathered}
\mathbf{k} \cdot \mathbf{G} \nu(\mathbf{G}) p_{0}+\sum_{\mathbf{G}^{\prime} \neq 0} \mathbf{G} \cdot \mathbf{G}^{\prime} \nu\left(\mathbf{G}-\mathbf{G}^{\prime}\right) p_{k}\left(\mathbf{G}^{\prime}\right)=0, \\
\left(k^{2} \overline{\boldsymbol{\nu}}-\omega^{2} \bar{\gamma}\right) p_{0}+\sum_{\mathbf{G}^{\prime} \neq 0} \mathbf{k} \cdot \mathbf{G}^{\prime} \nu\left(-\mathbf{G}^{\prime}\right) p_{k}\left(\mathbf{G}^{\prime}\right)=0 .
\end{gathered}
$$

Here $\bar{\nu} \equiv \nu(\mathbf{G}=0)$ and $\bar{\gamma} \equiv \gamma(\mathbf{G}=0)$ are the bulk average of $1 / \rho(\mathbf{r})$ and $1 / \lambda(\mathbf{r})$, respectively. For a binary composite $\bar{\nu}=f / \rho_{a}+(1-f) / \rho_{b}$, where $f$ is the filling fraction of the inclusions (material $a$ ). Note that the quadratic approximation, Eq. (7), involves only the terms with $\mathbf{G}=0$. Eliminating $p_{0}$ from Eqs. (6) and (7) and using the definition (5) we come to a homogenized set of equations valid in the limit $k, \omega \rightarrow 0$

$$
\begin{aligned}
& \left(c_{\mathrm{eff}}^{2} \bar{\gamma}-\bar{\nu}\right) \sum_{\mathbf{G}^{\prime} \neq 0} \mathbf{G} \cdot \mathbf{G}^{\prime} \nu\left(\mathbf{G}-\mathbf{G}^{\prime}\right) p_{k}\left(\mathbf{G}^{\prime}\right)+ \\
& \sum_{\mathbf{G}^{\prime} \neq 0}(\hat{\mathbf{k}} \cdot \mathbf{G})\left(\hat{\mathbf{k}} \cdot \mathbf{G}^{\prime}\right) \nu(\mathbf{G}) \nu\left(-\mathbf{G}^{\prime}\right) p_{k}\left(\mathbf{G}^{\prime}\right)=0 .
\end{aligned}
$$

The set of Eqs. (8) has nonzero solution if its determinant vanishes,

$$
\begin{aligned}
\underset{\mathbf{G}, \mathbf{G}^{\prime} \neq 0}{\operatorname{det}}\left[\left(c_{\mathrm{eff}}^{2} \bar{\gamma}-\bar{\nu}\right) \mathbf{G} \cdot \mathbf{G}^{\prime} \nu\left(\mathbf{G}-\mathbf{G}^{\prime}\right)+\right. \\
\left.(\hat{\mathbf{k}} \cdot \mathbf{G})\left(\hat{\mathbf{k}} \cdot \mathbf{G}^{\prime}\right) \nu(\mathbf{G}) \nu\left(-\mathbf{G}^{\prime}\right)\right]=0 .
\end{aligned}
$$

Although Eq. (9) is an infinite-order polynomial equation with respect to $\Lambda=\left(c_{\text {eff }}^{2} \bar{\gamma}-\bar{\nu}\right)$, it turns out that it has only a single nonzero solution. To obtain this solution we reduce Eq. (9) to a standard eigenvalue problem by multiplying it by the determinant of the inverse matrix, $\operatorname{det}\left\{\left[\mathbf{G} \cdot \mathbf{G}^{\prime} \nu\left(\mathbf{G}-\mathbf{G}^{\prime}\right)\right]^{-1}\right\}$.

$$
\underset{\mathbf{G}, \mathbf{G}^{\prime} \neq 0}{\operatorname{det}}\left[B\left(\mathbf{G}, \mathbf{G}^{\prime}\right)-\Lambda \delta_{\mathbf{G G}^{\prime}}\right]=0 .
$$

Here

$$
\begin{aligned}
B\left(\mathbf{G}, \mathbf{G}^{\prime}\right)=-\hat{\mathbf{k}} \cdot \mathbf{G} \nu(\mathbf{G}) \sum_{\mathbf{G}^{\prime \prime} \neq 0} & \hat{\mathbf{k}} \cdot \mathbf{G}^{\prime \prime} \nu\left(-\mathbf{G}^{\prime \prime}\right) \\
& \times\left[\mathbf{G}^{\prime \prime} \cdot \mathbf{G}^{\prime} \nu\left(\mathbf{G}^{\prime \prime}-\mathbf{G}^{\prime}\right)\right]^{-1} .
\end{aligned}
$$

The matrix $B\left(\mathbf{G}, \mathbf{G}^{\prime}\right)$ is written as a product of two factors, one of which depends only on $\mathbf{G}$ and the other only on $\mathbf{G}^{\prime}$. Then this matrix corresponds to a projection operator that has an eigenvalue $\Lambda=\operatorname{Tr} B\left(\mathbf{G}, \mathbf{G}^{\prime}\right)$. This results in the final formula for the effective speed of sound,

$$
\begin{array}{r}
c_{\mathrm{eff}}^{2}(\hat{\mathbf{k}})=\frac{\bar{\nu}}{\bar{\gamma}}-\frac{1}{\bar{\gamma}} \sum_{\mathbf{G}, \mathbf{G}^{\prime} \neq 0}(\hat{\mathbf{k}} \cdot \mathbf{G})\left(\hat{\mathbf{k}} \cdot \mathbf{G}^{\prime}\right) \nu(\mathbf{G}) \nu\left(-\mathbf{G}^{\prime}\right) \\
\times\left[\mathbf{G} \cdot \mathbf{G}^{\prime} \nu\left(\mathbf{G}-\mathbf{G}^{\prime}\right)\right]^{-1} .
\end{array}
$$

The effective speed of sound depends on the direction of propagation, and is valid for an arbitrary form of the unit cell, geometry of the inclusions, and material composition of the (liquid) phononic crystal. Equation (12) can be rewritten in the form

$$
c_{\mathrm{eff}}^{2}(\hat{\mathbf{k}})=A_{i j} \hat{k}_{i} \hat{k}_{j}, \quad i, j=x, y, z,
$$

where

$$
\begin{aligned}
A_{i j}=\frac{\bar{\nu}}{\bar{\gamma}} \delta_{i j}-\frac{1}{2 \bar{\gamma}} \sum_{\mathbf{G}, \mathbf{G}^{\prime} \neq 0}\left(G_{i} G_{j}^{\prime}+G_{j} G_{i}^{\prime}\right) \nu(\mathbf{G}) \nu\left(-\mathbf{G}^{\prime}\right) \\
\times\left[\mathbf{G} \cdot \mathbf{G}^{\prime} \nu\left(\mathbf{G}-\mathbf{G}^{\prime}\right)\right]^{-1} .
\end{aligned}
$$

It follows from Eq. (13) that radius vector $1 / c_{\text {eff }}(\hat{\mathbf{k}})$ sweeps an ellipsoid with semiaxes $1 / A_{x}, 1 / A_{y}$, and $1 / A_{z}$, where $A_{i}$ are the principal values of the tensor $A_{i j}$. Thus, the three constants, $A_{x}, A_{y}$, and $A_{z}$, determine completely the acoustic properties of a phononic crystal in the long-wavelength limit. The crystal is isotropic only if $A_{x}=A_{y}=A_{z}$; otherwise it exhibits anisotropy.

For the case of binary composites Eq. (12) supports the widely used Wood's law [9] $c_{\text {eff }}=\left(\lambda_{\text {eff }} / \rho_{\text {eff }}\right)^{1 / 2}$ since the dependence on the bulk moduli of the materials enters only through the parameter $1 / \lambda_{\text {eff }}=\bar{\gamma}=f / \lambda_{a}+$ $(1-f) / \lambda_{b}$. All the details about the microstructure enter through $\rho_{\text {eff }}$. Recently an excellent approximation for $\rho_{\text {eff }}$ was obtained in Ref. [14] for a random distribution of air bubbles in water. In order to compare the results for regular and random distribution of spherical bubbles we plot in Fig. $1 c_{\text {eff }}$ vs $f$ in a simple cubic lattice (solid dots). This phononic crystal is isotropic. The solid line shows the dependence obtained in Ref. [14] in the coherent potential approximation [15]. One can see that for small and moderate filling fractions $(f<0.3)$ the 


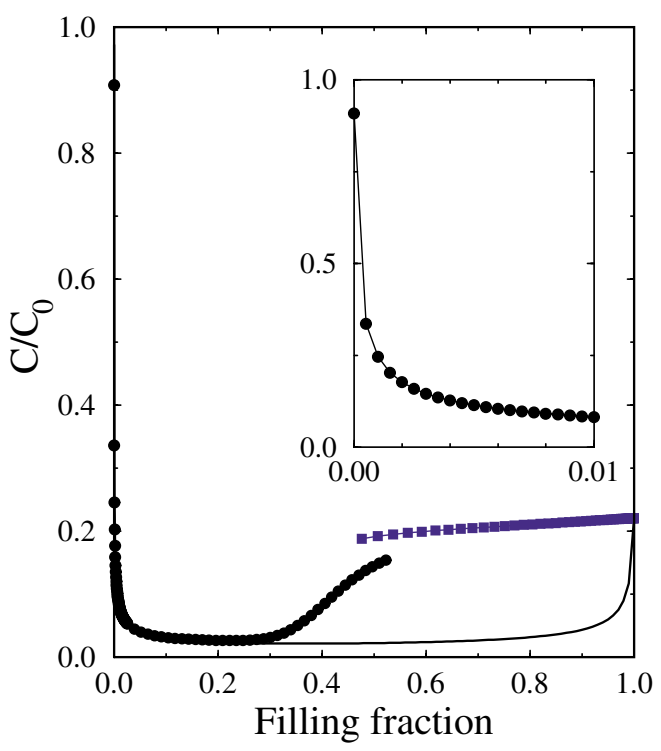

FIG. 1 (color online). Effective speed of sound calculated from Eq. (12) in a simple cubic lattice of air bubbles, $c_{a}=$ $330 \mathrm{~m} / \mathrm{s}$, in water, $c_{0}=1500 \mathrm{~m} / \mathrm{s}$, (circles) and in a conjugate lattice of water drops in air (squares) vs filling fraction of the air. The number of $\mathbf{G}$ values (plane waves) involved in these calculations is $N=800$ that guarantees a good convergence of the numerical results. Solid line shows the speed of sound obtained in Ref. [14]. Insert shows blowup of a region of low concentrations of the air.

coherent potential approximation gives the results that are very close to the exact formula (12). In particular, the fast drop of speed of sound for dilute mixtures $(f<0.02)$ is equally described by the two approaches. Direct numerical solution of the wave equation also gives similar results [16]. For $f>0.3$ the curve obtained from Eq. (12) grows up rapidly, and at the filling fraction $f_{c}=\pi / 6$ when spheres touch each other $c_{\text {eff }}$ reaches the value of $0.18 c_{0}$. It exceeds by almost ten times the value of $c_{\text {eff }}=$ $0.02 c_{0}$ obtained from the coherent potential approximation [14]. This rapid growth is due to an open air channel in a phononic crystal with overlapping spheres $\left(f>f_{c}\right)$ where sound propagates mostly through air. Therefore in a lattice of overlapping spheres $c_{\text {eff }}$ is close to the speed of sound in pure air, $c_{a}=0.22 c_{0}$. Since for a given structure $c_{\text {eff }}(f)$ is a continuous function, there is a transition region from a low-speed region at small filling fractions $(f<$ 0.3 ) to a high-speed region at $f$ close to $f_{c}$. Approximate theories fail to explain the region close to $f_{c}$. Here the interaction between bubbles becomes very strong, giving rise to a fast increase of the speed of sound.

In a conjugate lattice - water drops in air - the presence of water inclusions does not play a significant role. Squares in Fig. 1 show the effective speed of sound in a conjugate lattice. Water drops in air can be considered as rigid spheres where sound does not penetrate. It was demonstrated in Ref. [10] that the presence of rigid spheres decreases slightly the speed of sound in a matrix material. This tendency is clearly seen in Fig. 1. Note that the curves for the two lattices never meet each other because these two structures are not equivalent. However, for a special case of cubic inclusions the two lattices become geometrically equivalent at $f=0.5$. Therefore at this point the two curves meet each other; see Fig. 2. Note that for cubic atoms the transition region starts earlier, at $f \approx 0.12$, and at $f \approx 0.2$ the effective speed of sound practically saturates, reaching the value of $c_{a}$. Although the structure with cubic inclusions is not of a real situation, it helps to clarify that the fast increase of the speed of sound in the transition region for the case of noncubic inclusions is a manifestation of the continuity of the curve in Fig. 2. The latter is due to an additional symmetry between the direct and conjugate lattices.

Now we proceed with homogenization of solid twodimensional phononic crystals. We consider a uniform along $z$ elastic material with periodic (in the $x-y$ plane) dependence of the elastic moduli. Two elastic eigenmodes with transverse and mixed polarization can propagate in this system [3]. Here we consider only the transverse mode when the Bloch vector is in the $x-y$ plane and the displacement vector $\mathbf{u}=(0,0, u)$ is parallel to axis $z$. The dynamical equation for this polarization has the following form (see, e.g., [3]):

$$
\rho(\mathbf{r}) \frac{\partial^{2} u}{\partial t^{2}}=\nabla_{t} \cdot\left(\tau(\mathbf{r}) \nabla_{t} u\right)
$$

Here $\nabla_{t}=(\partial / \partial x, \partial / \partial y, 0)$ is two-dimensional gradient. Since the vibrations are transversal, only the shear modulus $\tau$ enters in the wave Eq. (15). A comparison with Eq. (1) shows that Eq. (15) is a two-dimensional version of Eq. (1) provided that $1 / \rho=\nu$ is replaced by $\tau$ and $1 / \lambda=\gamma$ is replaced by $\rho$. After these substitutions we can write the formula for the speed of transversal sound directly from Eq. (12),

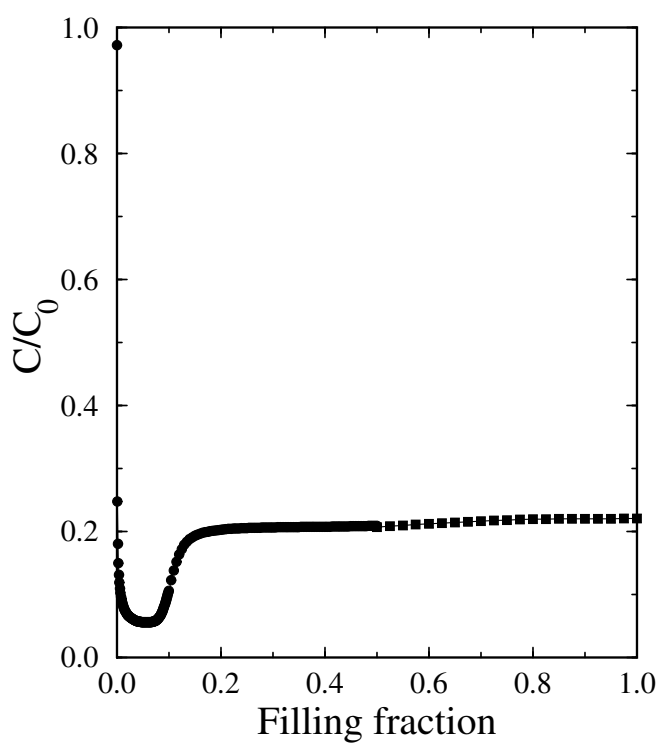

FIG. 2. Same as in Fig. 1 but for cubic form of inclusions. 


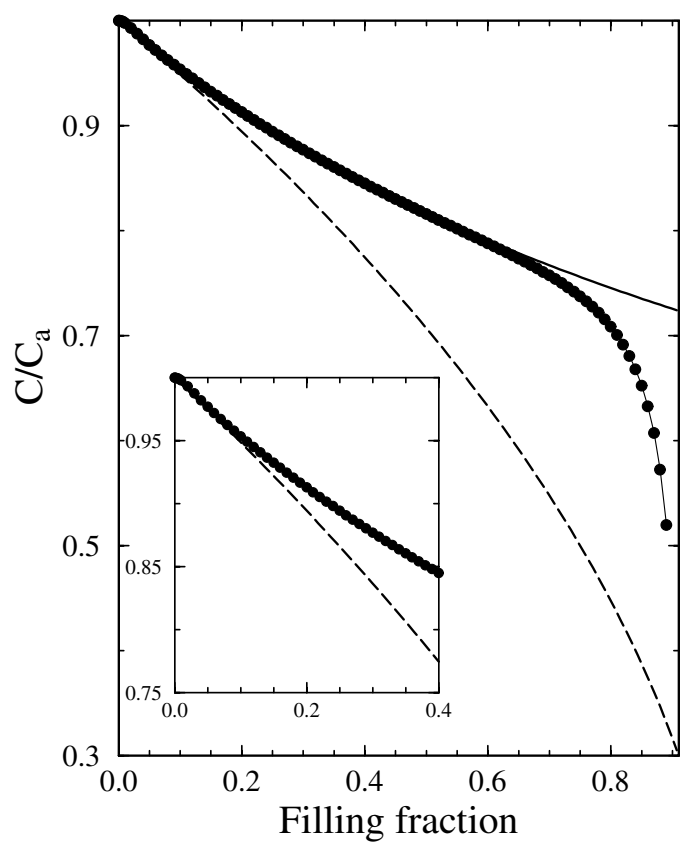

FIG. 3. Circles represent the speed of sound calculated for acoustic lens of Ref. [5]. Because of the presence of the thirdorder rotational axis of symmetry this phononic crystal is isotropic.

$$
\begin{array}{r}
c_{\mathrm{eff}}^{2}(\hat{\mathbf{k}})=\frac{\bar{\tau}}{\bar{\rho}}-\frac{1}{\bar{\rho}} \sum_{\mathbf{G}, \mathbf{G}^{\prime} \neq 0}(\hat{\mathbf{k}} \cdot \mathbf{G})\left(\hat{\mathbf{k}} \cdot \mathbf{G}^{\prime}\right) \tau(\mathbf{G}) \tau\left(-\mathbf{G}^{\prime}\right) \\
\times\left[\mathbf{G} \cdot \mathbf{G}^{\prime} \tau\left(\mathbf{G}-\mathbf{G}^{\prime}\right)\right]^{-1} .
\end{array}
$$

Here $\mathbf{G}=\left(G_{x}, G_{y}, 0\right)$ are two-dimensional reciprocal lattice vectors. For transversal vibrations the Wood's law is not applicable. One can see from Eq. (16) that the effective speed of sound is determined by the average density $\bar{\rho}$ and the effective shear modulus depends on the details of the microstructure.

In the experiment [5] with focusing an acoustic beam by a phononic crystal of aluminum cylinders the transversal vibrations were not excited since between the cylinders sound propagates through the air. Because of very high contrast between the acoustic impedances of aluminum and air, the cylinders can be considered as rigid. In this case, a two-dimensional version of Eq. (12) is applicable with $\nu_{\mathrm{Al}}=\gamma_{\mathrm{Al}}=0$. The results of calculations for a hexagonal lattice are shown in Fig. 3. They are in reasonable agreement with experimental data [5] and with a simple model $c_{\text {eff }}=c_{a} / \sqrt{1+f}$ proposed there (solid line in Fig. 3). Unlike this, the approximation $c_{\mathrm{eff}}=c_{a} \sqrt{1-f}$ proposed in Ref. [17] (dashed line in Fig. 3) strongly deviates from the exact result.

In conclusion, we report exact analytical results for the speed of longitudinal sound in 3D periodic gas-liquid mixtures and for the speed of transversal sound in a periodic arrangement of solid cylinders in a solid matrix
(2D phononic crystal). Our formulas reproduce the drastic drop of the speed of sound in water with air bubbles. We demonstrate that the phenomenological Wood's law holds for the longitudinal sound but it fails for the transversal modes.

This work was supported by CONACyT (Mexico) Grants No. 42136-F and No. 33808-E. This work has been completed at ICTP (Italy). A. A. K. is thankful to the ICTP Condensed Matter Group for useful discussions.

[1] Lord Rayleigh, Theory of Sound (Dover, New York, 1945); P. M. Morse and K. U. Ingaard, Theoretical Acoustic (McGraw-Hill, New York, 1968).

[2] J.V. Sánchez-Pérez et al., Phys. Rev. Lett. 80, 5325 (1998); F. R. Montero de Espinosa, E. Jiménez, and M. Torres, Phys. Rev. Lett. 80, 1208 (1998).

[3] M. S. Kushwaha, P. Halevi, L. Dobrzynski, and B. Djafari-Rouhani, Phys. Rev. Lett. 71, 2022 (1993); M. M. Sigalas and E. N. Economou, Solid State Commun. 86, 141 (1993); M. S. Kushwaha, Int. J. Mod. Phys. B 10, 977 (1996).

[4] M. Torres, F. R. Montero de Espinosa, D. García-Pablos, and N. García, Phys. Rev. Lett. 82, 3054 (1999); M. Torres, F. R. Montero de Espinosa, and J. L. Aragón, Phys. Rev. Lett. 86, 4282 (2001).

[5] F. Cervera et al., Phys. Rev. Lett. 88, 023902 (2002).

[6] Gail ter Haar, Phys. Today 54, No. 12, 29 (2001).

[7] A. Bensoussan, J.-L. Lions, and G. Papanicolau, Asymptotic Analysis for Periodic Structures (NorthHolland, Amsterdam, 1978); N. S. Bakhvalov and G. P. Panasenko, Homogenization. Averaging Process in Periodic Media. Mathematical Problems in the Mechanics of Composite Materials (Kluwer, New York, 1989).

[8] M. Birman and T. Suslina, Operator Theory Adv. Appl. 129, 71 (2001).

[9] A.W. Wood, Textbook of Sound (Macmillan, New York, 1941).

[10] D. Bai and J. B. Keller, J. Acoust. Soc. Am. 82, 1436 (1987).

[11] P. Sheng, in Homogenization and Effective Moduli of Materials and Media, edited by J. L. Ericksen, D. Kinderlehrer, R. Khon, and J.-L. Lions (Springer-Verlag, New York, 1986), p. 196.

[12] Z. Hashin and S. Shtrickman, J. Mech. Phys. Solids 10, 335 (1962); L. A. Rubenfeld and J. B. Keller, SIAM J. Appl. Math. 17, 495 (1969).

[13] P. Halevi, A. A. Krokhin, and J. Arriaga, Phys. Rev. Lett. 82, 719 (1999); A. A. Krokhin, P. Halevi, and J. Arriaga, Phys. Rev. B 65, 115208 (2002).

[14] M. Kafesaki, R. S. Penciu, and E. N. Economou, Phys. Rev. Lett. 84, 6050 (2000).

[15] M. Kafesaki and E. N. Economou, Phys. Rev. B 60, 11993 (1999).

[16] A. A. Ruffa, J. Acoust. Soc. Am. 91, 1 (1992).

[17] B. C. Gupta and Zh. Ye, Phys. Rev. E 67, 036603 (2003). 